\title{
Characterization of Full-Range Time Evolution in Active Thermography
}

\author{
by S. Shepard, M. Frendberg and Y. Hou
}

Thermal Wave Imaging, Inc., 845 Livernois, Ferndale MI, 48220 USA, sshepard@thermalwave.com

\begin{abstract}
The depth range of active thermography is limited by several factors including the amount of excitation applied to the sample and the noise level of the IR camera. However, the subsurface diffusion process continues, regardless of our ability to monitor it. On a sufficiently large time scale the surface temperature response appears in the logarithmic domain as a transition from an initial 1-dimensional diffusion state to a final one, with the beginning and end of the transition indicated by a peak in the $2^{\text {nd }}$ derivative. The lifetime of transient diffusion process, during which the usual contrast and peak slope maxima are observed, can be defined as the ratio of the times at which the $2^{\text {nd }}$ derivative maxima occur.
\end{abstract}

\section{Introduction}

Analysis of active thermography data has traditionally been discussed in terms of contrast between a point on the sample surface associated with an internal flaw, and an intact background point or reference standard. Detectability of a flaw is often expressed in terms of the maximum flaw-background contrast amplitude, and the time scale for detection may be estimated from the time at which the maximum contrast occurs. Contrast amplitude depends on the flaw depth, diameter and composition, as well as the excitation energy applied to the sample surface. The introduction of the Thermographic Signal Reconstruction (TSR) method in 2001 shifted attention from contrast methods to single pixel analysis based on logarithmic temperature vs. time behavior, and its derivatives. Unlike contrast methods, the TSR approach can be applied to both spatially discrete features and extended interfaces. The TSR $1^{\text {st }}$ and $2^{\text {nd }}$ derivatives scale the signal to a fixed range that is invariant with respect to excitation energy, and in which anomalies in 1 dimensional diffusion are apparent without need for a flaw free reference [1].

In principle, it is possible to apply an unlimited amount of energy to a sample in order to interrogate any thickness or material configuration. In practice, however, the depth range of active thermography is limited by several factors including a) the amount of excitation energy that can realistically be applied to the sample nondestructively; b) the dynamic range and noise level of the IR camera; and c) the amount of time one is willing to commit to monitoring the surface temperature response of the sample. We have shown in previous work that the limit of detectability of a subsurface interface is a function of the ratio of excitation energy to camera noise equivalent temperature difference, and also the thermal effusivity of the sample [2]. Once we have reached this limit, changes in surface temperature are too small to be detected by the IR camera, and are likely to be masked by background radiation in a real experiment. Thus, a feasible thermography application is one in which the depth plane of interest can be interrogated before this extinction limit is reached. However, the subsurface diffusion process continues, despite our inability to monitor it.

\section{Lifetime of the Transient Diffusion Process}

On a sufficiently large time scale the active thermography process can be viewed as a transition from 1-dimensional steady state diffusion before incident heat encounters a subsurface interface to a second asymptotic steady state. For the purposes of NDT, it is normally the transition between these 1-dimensional diffusion states that yields information about the subsurface structure of a sample (the usual events of interest in active thermography, e.g. the peak contrast, peak slope, and logarithmic $2^{\text {nd }}$ derivative maximum are all transient events that occur during this transition). However, it is instructive to consider the surface temperature response of active thermography process over the complete diffusion cycle, i.e. from the initial to the final 1-dimensional steady state. As an example, we consider the case of a flat bottom hole in an infinitely thick slab, using data generated using finite difference modeling (Fig. 1).

In viewing the extended signal and its derivatives in the logarithmic domain, we observe the usual maxima in the contrast, slope and $2^{\text {nd }}$ derivative $\left(t^{\star}\right)$, and note that these occur at progressively earlier times, consistent with the so-called "precession" of events observed elsewhere [3]. We also observe a later event in the $2^{\text {nd }}$ derivative, a larger peak that occurs at time $t^{\star \star}$, indicating the asymptotic return to 1-dimensional diffusion. We can consider the "lifetime" of the transient diffusion process to be the ratio $t^{* *} / t^{*}$. While the specific amplitudes and times associated with these 4 events will vary based on the size and depth of the hole and the sample material, the overall shape and polarity of the signals will apply to any flat bottom hole case. 


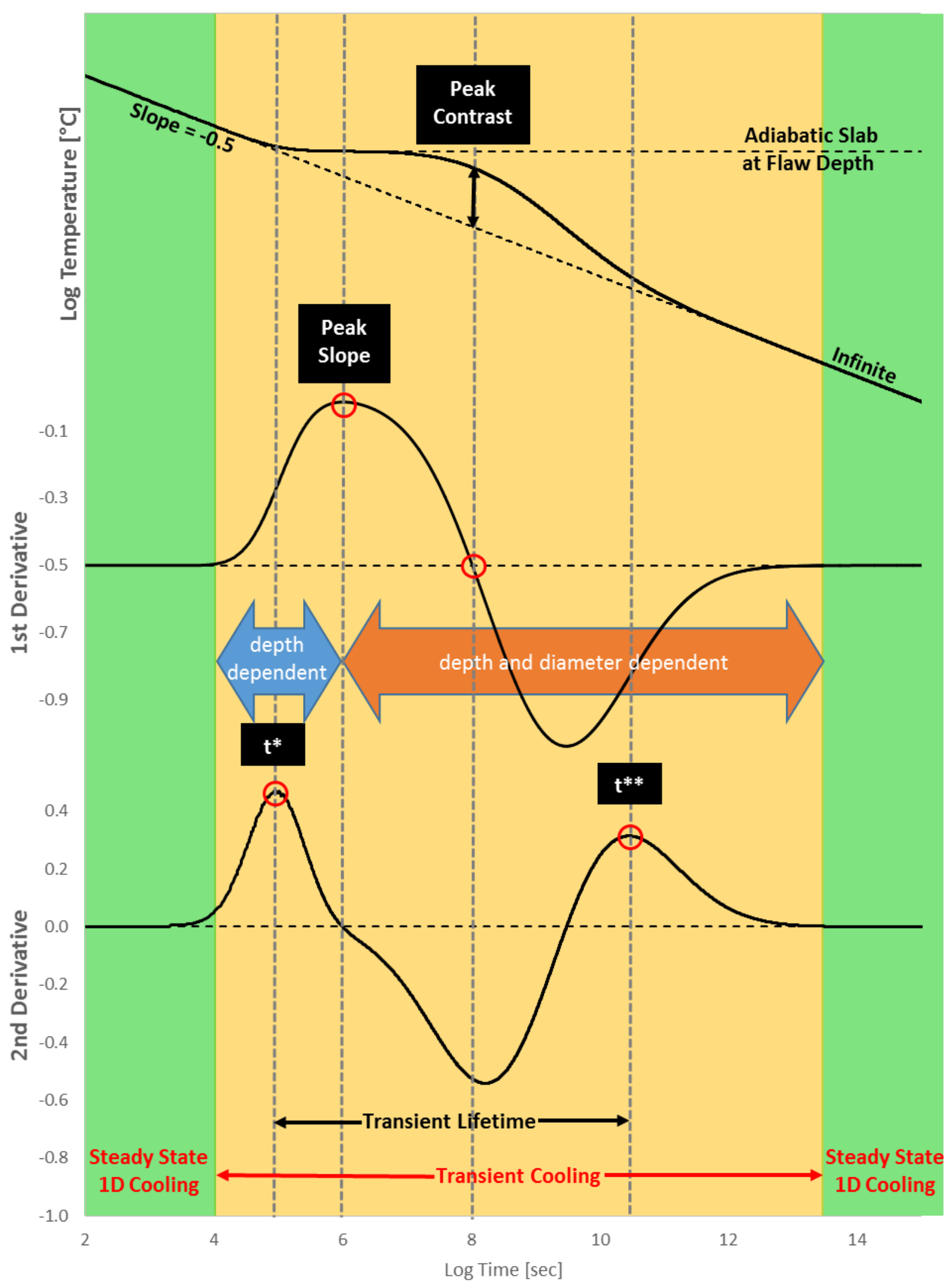

Figure 1. The logarithmic temperature (top), $1^{\text {st }}$ derivative (center) and $2^{\text {nd }}$ derivative (bottom) vs. time of the surface temperature above the center point of a flat bottom hole in an infinitely thick sample. 


\section{REFERENCES}

[1] Shepard S., Lhota J., Rubadeux B., Wang D. and Ahmed T., "Reconstruction and Enhancement of Active Thermographic Image Sequences," Optical Engineering, 42(5):1337-1342, 2003

[2] Shepard S., Lhota, J. and Ahmed, T., "Flash thermography contrast model based on IR camera noise characteristics". Nondestructive Testing and Evaluation, Volume 22, 2-3, pp. 113-126, 2007

[3] Balageas, D.L., "Defense and illustration of time-resolved pulsed thermography for NDE", Quantitative InfraRed Thermography, Vol. 9, No. 1, June 2012, 3-32 